\title{
Alteration of the intestinal barrier and GLP2 secretion in Berberine-treated type 2 diabetic rats
}

\author{
C Y Shan*, J H Yang*, Y Kong, X Y Wang, M Y Zheng, Y G Xu, Y Wang, H Z Ren, \\ B C Chang and L M Chen
}

Key Laboratory of Hormone and Development (Ministry of Health), Metabolic Disease Hospital and Tianjin Institute of Endocrinology, Tianjin Medical University, Tianjin 300070, China

*(C Y Shan and J H Yang contributed equally to this work)

\begin{abstract}
For centuries, Berberine has been used in the treatment of enteritis in China, and it is also known to have anti-hyperglycemic effects in type 2 diabetic patients. However, as Berberine is insoluble and rarely absorbed in gastrointestinal tract, the mechanism by which it works is unclear. We hypothesized that it may act locally by ameliorating intestinal barrier abnormalities and endotoxemia. A high-fat diet combined with low-dose streptozotocin was used to induce type 2 diabetes in male Sprague Dawley rats. Berberine $(100 \mathrm{mg} / \mathrm{kg})$ was administered by lavage to diabetic rats for 2 weeks and saline was given to controls. Hyperinsulinemia and insulin resistance improved in the Berberine group, although there was no significant decrease in blood glucose. Berberine treatment also led to a notable restoration of intestinal villi/mucosa structure and less infiltration of inflammatory cells, along with a decrease in plasma lipopolysaccharide (LPS) level. Tight junction protein zonula occludens 1 (ZO1) was also decreased in diabetic rats but was restored by Berberine treatment. Glutamine-induced glucagon-like peptide 2 (GLP2) secretion from ileal tissue decreased dramatically in the diabetic group but was restored by Berberine treatment. Fasting insulin, insulin resistance index, plasma LPS level, and ZO1 expression were significantly correlated with GLP2 level. In type 2 diabetic rats, Berberine treatment not only augments GLP2 secretion and improves diabetes but is also effective in repairing the damaged intestinal mucosa, restoring intestinal permeability, and improving endotoxemia. Whether these effects are mechanistically related will require further studies, but they certainly support the hypothesis that Berberine acts via modulation of intestinal function.
\end{abstract}

Correspondence should be addressed to B C Chang or L M Chen Email changbc1970@126.com or xfx22081@vip.163.com

\section{Introduction}

According to the World Health Organization (WHO), an estimated 220 million people globally are suffering from diabetes mellitus (Whiting et al. 2011). Without further actions or interventions, this number is likely to double by the year 2030. Because it is unlikely that there has been a dramatic alteration in genetic factors in the past decades,
Key Words
- berberine
- type 2 diabetes
- GLP2
- intestinal barrier

environmental factors must play a key role in the rapid rise in diabetes. Gut microbiota has been proposed as a key player (Burcelin et al. 2009) with populations showing marked differences between healthy, obese, and type 2 diabetic patients (Bäckhed et al. 2004, Qin et al. 2012). The dysbiosis of gut microbiota (at phyla, genus, or species 
level) has the potential to affect host metabolism and energy storage (Ley et al. 2006) and to affect gut permeability and, as a consequence, give rise to metabolic endotoxemia and higher plasma lipopolysaccharide (LPS). In addition, gut peptides such as glucagon-like peptide 1 (GLP1) and GLP2 may play key roles in these processes (Tremaroli \& Bäckhed 2012). For example, GLP2, which is secreted by intestine L cells, is a key regulator of intestinal permeability (Cani et al. 2009). Therapeutic regimes that target intestinal microbiota and intestinal barrier show a broad prospect in treating diabetes (Kootte et al. 2012). One such factor is Berberine, a natural plant alkaloid isolated from Chinese herb Coptis chinensis (Huanglian). It is commonly used for treating bacterial diarrhea and has recently been demonstrated to be clinically effective in alleviating type 2 diabetes (T2DM; Ley et al. 2006, Cani et al. 2008). As Berberine is poorly absorbed into the bloodstream from the gut (Han et al. 2011), the mechanism of its action is not clear, but modulation of gut microbiota and intestinal permeability are plausible possibilities that we wished to explore. Berberine stimulates GLP1 secretions (Yu et al. 2010), and because GLP1 is co-released with GLP2 from intestinal $\mathrm{L}$ cells, it is possible that Berberine may be able to modulate GLP2 secretion. Therefore, in this study, we investigated the alterations of intestinal barrier and GLP2 secretion before and after Berberine treatment in type 2 diabetic rats, with the intention of establishing an association between Berberine's antidiabetic effects and intestinal permeability, endotoxemia, and GLP2 secretion.

\section{Materials and methods}

\section{Animal and protocol}

T2DM was induced in male Sprague Dawley rats (7-8 weeks of age, 200-250 g) using streptozotocin (STZ) according to Bi et al. (2009). Briefly, the rats were fed ad libitum with a high-fat diet (63\% calories as fat), which was prepared by supplementing normal chow with $10 \%(\mathrm{w} / \mathrm{w})$ lard, 10\% (w/w) sucrose, $1 \%(\mathrm{w} / \mathrm{w})$, cholesterol, and $0.3 \% \mathrm{w} / \mathrm{w}$ bile acid (sodium salt) for 8 weeks. This was followed by a single low-dosage i.p. injection of STZ (30 mg/kg; Sigma) after 12 -h fast. A group of nondiabetic rats fed solely on chow diet was used as controls. Diabetic rats were divided into two subgroups: a vehicle-treated group $(n=11)$ and a Berberine-treated group $(n=11)$. Plasma glucose in diabetic rats increased to at least $16.7 \mathrm{mmol} / \mathrm{l}$ within 3 days after STZ injection and remained at least to this level throughout the experiment. Berberine intervention was initiated on the 7th day after STZ injection and was continued for 2 weeks. The Berberine solution was prepared in PBS and delivered by oral lavage at $100 \mathrm{mg} / \mathrm{kg}$ per day. The control group was given a similar volume of PBS as vehicle. Five rats died in the first 3 days after STZ injection, and two rats died (one in Berberine group and one in vehicle group) after Berberine or vehicle intervention.

Blood was drawn from the abdominal aorta under anesthesia (sodium pentobarbital, $40 \mathrm{mg} / \mathrm{kg}$ body weight; Sigma) for the measurement of LPS and insulin, and plasma was separated and stored at $-80^{\circ} \mathrm{C}$ until examined. Four centimeters of ileum from rats were excised and fixed in 4\% paraformaldehyde for hematoxylin and eosin (HE) staining and immunohistochemical analysis of zonula occludens 1 (ZO1). Another $3 \mathrm{~cm}$ of ileum were stored in PBS for the analysis of glutamineinduced GLP2 secretion. This study was approved by the Tianjin Medical University Animal Committee, and the rats were maintained in accordance with the Tianjin Medical University guidelines for the care and use of laboratory animals.

\section{Plasma insulin and LPS concentrations}

Fasting glucose levels in tail vein blood were assayed by an enzymatic method using a blood glucose meter (Johnson $\&$ Johnson Medical Ltd, New Brunswick, NJ, USA); fasting plasma insulin concentration was assayed by ELISA (Rat Insulin ELISA KIT, Cusabio, Barksdale, DE, USA). LPS concentration was assayed by ELISA (Rat LPS ELISA Kit). Homeostasis model assessment insulin resistance (HOMAIR) was calculated as an indicator of insulin resistance according to the formula: HOMA-IR=Fasting glucose $(\mathrm{mM}) \times$ Fasting insulin $(\mu \mathrm{U} / \mathrm{ml}) / 22.5$.

\section{Histology}

The ileum sample fixed in $4 \%$ paraformaldehyde was paraffin embedded, sectioned into $8 \mu \mathrm{m}$-thick slices, and stained with both HE dye and immunohistochemistry. For immunohistostaining of ZO1, paraffin-embedded sections were deparaffinized using xylene and re-hydrated in a series of alcohols, before incubation in antigen retrieval buffer (0.01 M citrate-buffered saline, $\mathrm{pH}$ 6.0) for $5 \mathrm{~min}$ at $95^{\circ} \mathrm{C}$ and then for $20 \mathrm{~min}$ at room temperature. The sections were then treated with $3 \%(\mathrm{v} / \mathrm{v})$ hydrogen peroxide for $5 \mathrm{~min}$. The slides were treated with $10 \%$ $(\mathrm{v} / \mathrm{v})$ normal goat serum to prevent nonspecific antibody binding. The samples were treated with ZO1 primary antibody (Wuhan Boster Biological Technology Co., Ltd,

Published by Bioscientifica Ltd 
Wuhan, China) at a dilution of 1:80 in PBS overnight at $4{ }^{\circ} \mathrm{C}$. Imaging was performed using a Nikon microscope and analyzed by HMIAS-2000 software (Championimage, Inc., China).

Three rats in each group were tested for ZO-1 expression. In each section of ileum, five different areas were selected for scoring. The staining score was analyzed by two different pathologists blinded to this study. The immunohistostaining was calculated by combining an estimate of the percentage of immunoreactive cells (quantity score) with an estimate of the staining intensity (staining intensity score). Staining intensity was rated on a scale of $0-3$, with $0=$ negative; $1=$ weak; $2=$ moderate, and $3=$ strong. When there was multifocal immunoreactivity and there were significant differences in staining intensities between foci, the average of the least intense and most intense staining was recorded. The raw data were converted to the immunohistostaining by multiplying the quantity and staining intensity scores (Soslow et al. 2000).

\section{Glutamine-induced secretion of GLP2 in ileum tissue}

Three centimeters of distal ileum were kept in PBS for the determination of glutamine-stimulated GLP2 secretion. In this procedure, the ileum was rinsed with PBS and cut open in a longitudinal direction. With the basolateral side upward, the outer muscle layers were carefully stripped off. After that, the ileum was cut into three segments, each $1 \mathrm{~cm}$ long. Then, each segment was chopped using eye scissors and transferred to a 24-well plate kept on ice and filled with $500 \mu \mathrm{l}$ PBS per well. The plate was brought up to room temperature and incubations were initiated by replacing the buffer solution with $500 \mu \mathrm{l}$ of pre-warmed 1640 cell culture medium (containing $2.05 \mathrm{mM}$ glutamine). The tissues were incubated for $30 \mathrm{~min}$ at $37^{\circ} \mathrm{C}$ in a humidified incubator at $5 \% \mathrm{CO}_{2}$ and then $4 \mathrm{mM}$ glutamine was added to each well to stimulate the secretion of GLP2 for another $30 \mathrm{~min}$. After removal of the tissue pieces, the conditioned medium was stored at $-70{ }^{\circ} \mathrm{C}$. GLP2 concentration in the medium was assayed by ELISA (Rat GLP2 ELISA Kit, Cusabio).

\section{Statistical analysis}

Results were presented as mean with the s.E.M. The statistical significance of differences was analyzed by one-way ANOVA followed by post hoc Bonferroni's multiple comparison tests or Kruskal-Wallis for nonparametric data followed by Dunn's multiple comparison tests. Multiple correlation analyses were assessed by the
Pearson's test. The analyses were performed using SPSS windows version 17.0 , and $P<0.05$ was considered to be statistically significant.

\section{Results}

\section{Plasma concentration of insulin, HOMA-IR, and LPS}

After 3 days of STZ injection, the plasma glucose in diabetic rats was increased greatly compared with normal rats $(24.0 \pm 3.1$ vs $5.1 \pm 0.3 \mathrm{mmol} / \mathrm{l}, P<0.01)$ and were stabilized at a steady high level after 7 days. Compared with normal rats, diabetic rats had a significantly lower body weight (344.0 \pm 49.9 vs $444.8 \pm 39.7 \mathrm{~g}, P<0.01)$ but higher blood glucose $(27.7 \pm 1.9$ vs $5.4 \pm 0.4 \mathrm{mmol} / \mathrm{l}, P<0.01)$, plasma insulin ( $42.34 \pm 6.31$ vs $18.11 \pm 4.36 \mu \mathrm{U} / \mathrm{ml}, P<0.01)$, and HOMA-IR $(54.59 \pm 13.93$ vs $4.15 \pm 1.01, P<0.01)$.

Blood glucose in the Berberine intervention group showed a trend to decrease after 7 days of intervention, and again after another 7 days of intervention (28.7 \pm 3.1 , $27.2 \pm 3.1$, and $26.9 \pm 2.2 \mathrm{mmol} / \mathrm{l})$, but these decreases were not significantly different from those in the T2DM group $(28.8 \pm 5.4,27.7 \pm 1.9$, and $28.2 \pm 4.2 \mathrm{mmol} / \mathrm{l})$.

Body weight was decreased in the first week after Berberine intervention, but then it increased greatly in the following week $(P<0.01)$; however, in the control group, the body weight increased steadily $(P<0.01)$, and in the vehicle treatment T2DM group, the body weight decreased steadily $(P<0.01)$, with the difference between the three groups being significant (Fig. 1).

In type 2 diabetic rats, the plasma level of LPS was significantly higher than that in normal rats, and this supports the existence of a serious endotoxemia in type 2 diabetic rats. After 2 weeks of Berberine treatment, plasma LPS levels decreased dramatically along with an improvement in hyperinsulinemia $(P<0.05)$ and insulin sensitivity $(P=0.061)$ (Fig. 2$)$.

\section{Structural change of ileum}

The ileum of diabetic rats showed significant structural changes compared with control rats. In diabetic rats, part of the villi and epithelial cells were detached, and the heights of the remaining villi were decreased. The interstitium of the ileum showed serious swelling and more inflammatory cell infiltration compared with control rats. Berberine was effective at reversing these changes and increased villi/mucosa height, reduced infiltration of inflammatory cell, and ameliorated detachment of villi/ epithelial cells (Fig. 3).

Published by Bioscientifica Ltd 

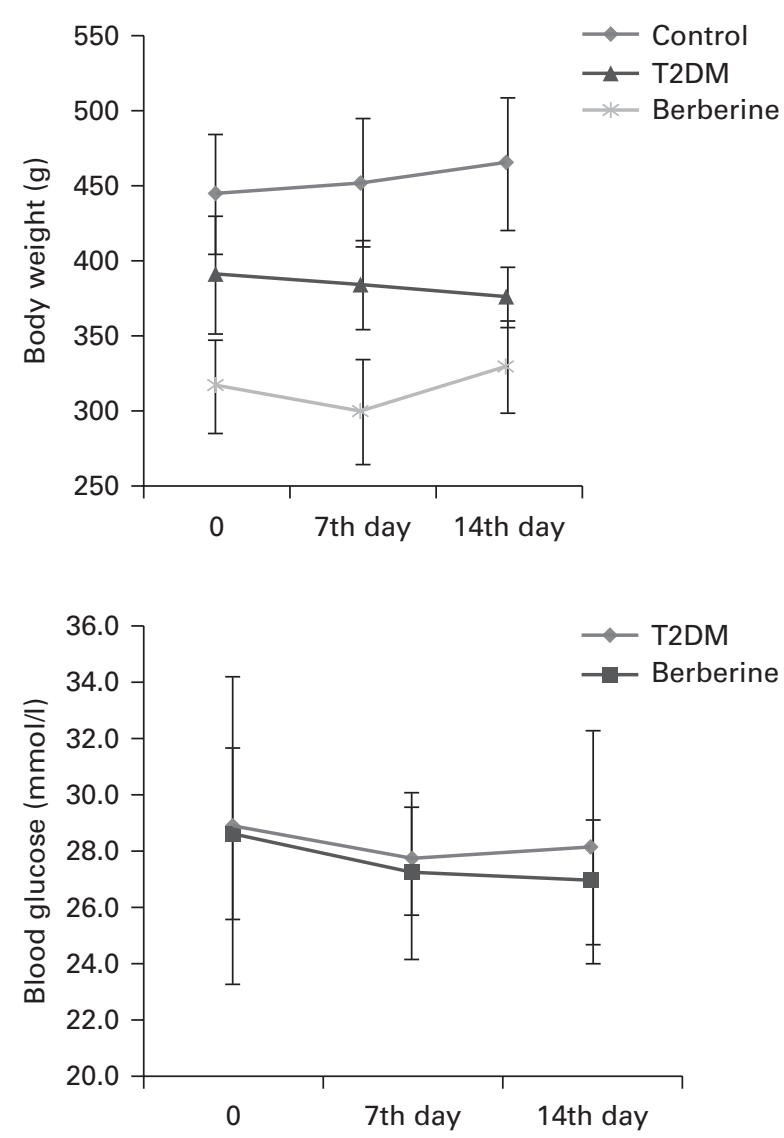

Figure 1

Alteration of body weight and blood glucose after Berberine intervention. Type 2 diabetic rats were treated with Berberine $(100 \mathrm{mg} / \mathrm{kg}$, Berberine group) or vehicle (T2DM group) for 2 weeks, and body weight and blood glucose were measured each week. Nondiabetic rats were used as control group. Differences between each group were significant for body weight $(P<0.05)$ but not for blood glucose. Full colour version of this figure available via http://dx.doi.org/10.1530/JOE-13-0184.

\section{Expression of intestinal tight junction protein}

In control rats, ZO1 staining strongly labeled the cytoplasm of enterocytes and gave rise to a well-defined and homogeneous distribution of label at the brush border level, leading to a well-defined thin line of staining.
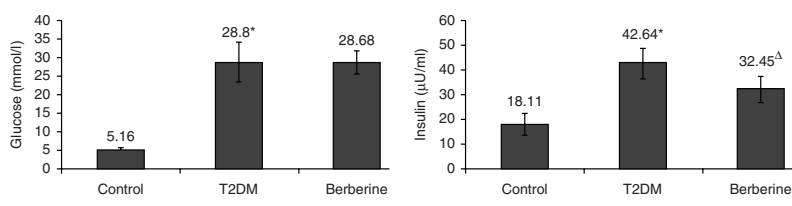

Figure 2

Plasma concentration of insulin, HOMA-IR, and LPS. Type 2 diabetic rats were treated with Berberine (100 mg/kg, Berberine group) or vehicle (T2DM group) for 2 weeks, and nondiabetic rats were used as control
However, in diabetic rats, the staining was not only at a lower level but also extremely irregular or absent at the luminal surface of the intestinal epithelium. In Berberinetreated rats, cytoplasmic staining was strong and the brush borderline appeared similar to healthy mucosa (Fig. 4).

\section{GLP2 secretion induced by glutamine in ileum tissue}

Compared with the control group, glutamine-stimulated GLP2 secretion was decreased in type 2 diabetic rats but was restored after 14 days of intervention with Berberine. Pearson correlation analysis revealed that fasting insulin level, HOMA-IR index, plasma LPS level, and ZO1 expression were significantly related to plasma GLP2 level; the Pearson correlations were $r=-0.723$ $(P=0.012), r=-0.641 \quad(P=0.034), r=-0.630 \quad(P=0.025)$, and $r=0.819(P=0.007)$ respectively (Fig. 5).

\section{Discussion}

Association between inflammation and T2DM originated in 1959 when epidemiological studies showed a rise in acute-phase response proteins in serum of type 2 diabetic patients compared with controls (Fearnley et al. 1959, Ogston \& McAndrew 1964). Later, a specific link between inflammatory and metabolic responses was made with the discovery that compared with lean tissue, obese adipose tissue secretes inflammatory cytokines and that these inflammatory cytokines themselves can inhibit insulin signaling (Hotamisligil et al. 1996). The definitive proof of such a connection between inflammatory mediators and insulin resistance in obesity and T2DM came from genetic studies that interfered with inflammatory mediators and demonstrated beneficial effects of this interference on insulin action (Uysal et al. 1997). The important role of inflammation in T2DM is further proved recently by Goldfine who has found that salsalate therapy lowered fasting glucose, increased adiponectin, and reduced adipose tissue NF- $\mathrm{KB}$ activity in persons with abnormal

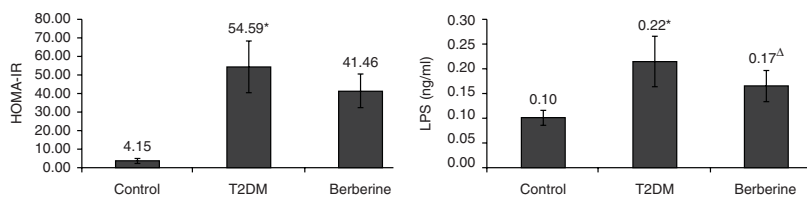

group. Blood glucose, plasma insulin, and plasma LPS concentrations were measured; *compared with control group $P<0.05 ;{ }^{\Delta}$ compared with type 2 diabetic group $P<0.05$.

Published by Bioscientifica Ltd. 

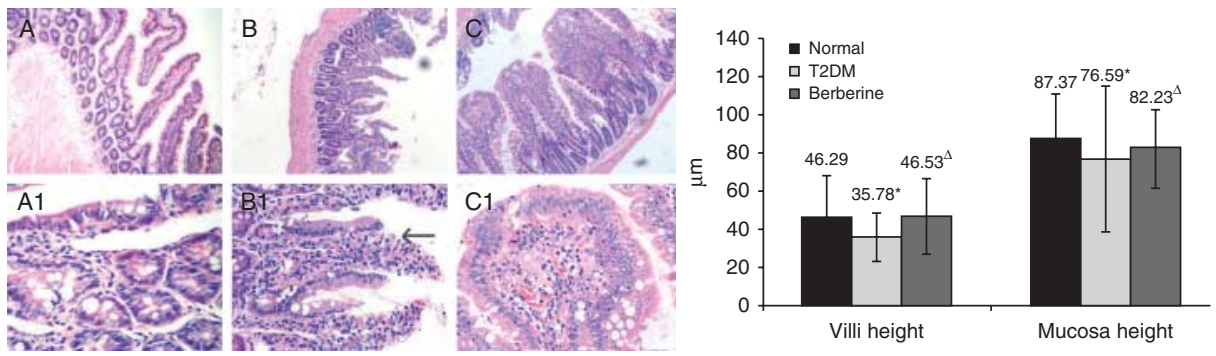

Figure 3

Structure alteration in type 2 diabetic rats and control rats. Type 2 diabetic rats were treated with Berberine (100 mg/kg, Berberine group) or vehicle (T2DM group) for 2 weeks, and nondiabetic rats were used as control group. Villi height and mucosa height were measured. In diabetic rats, the mucosa were damaged and incomplete with epithelial cell detached (arrow in B1); the villi height and mucosa height were decreased in diabetic rats and were improved in Berberine rats; $(A)$ control rats $\mathrm{HE} \times 10$;

(A1) control rats $\mathrm{HE} \times 40$; (B) T2DM rats $\mathrm{HE} \times 10$; (B1) T2DM rats $\mathrm{HE} \times 40$; (C) Berberine rats $\mathrm{HE} \times 10$; (C1) Berberine rats $\mathrm{HE} \times 40$. * Compared with control group $P<0.05 ;{ }^{\Delta}$ Compared with type 2 diabetic group $P<0.05$. glucose tolerance (Goldfine et al. 2013). Despite this, the origin of the inflammation is unclear. One intriguing possibility is that alterations in intestinal microbiota and function may be responsible. Indeed, changes in gut microbiota and increased intestinal permeability can induce metabolic inflammation by inducing endotoxemia (Cani et al. 2008, 2009). It has been proved that, antibiotics can improve glucose metabolism in diabetics (Chou et al. 2008, Membrez et al. 2008), and this is associated with modified cecal microbiota profile (Chou et al. 2008). Similarly, norfloxacin and ampicillin suppression of cecal aerobic and anaerobic bacteria in $o b / o b$ mice was accompanied by a significant improvement in fasting glycemia and oral glucose tolerance. Simultaneously, plasma LPS and tumor necrosis factor- $\alpha$ levels were decreased in the antibiotic-treated mice, suggesting that modulating gut microbiota ameliorated the inflammatory status in the intestine of the animals (Membrez et al. 2008).

Berberine, one of the main constituents of Rhizoma coptidis, is often used to treat bacterial diarrhea in China (Yan et al. 2011). In 1988, the glucose-lowering effect of
Berberine in diabetic patients was reported (Ni 1988). Since then, there have been many studies that confirmed Berberine's antidiabetic effect. For example, Yin et al. (2008) found that diabetic patients that took $500 \mathrm{mg}$ Berberine three times daily for 13 weeks showed decreased HbA1c levels (from 9.5 to $7.5 \%$ ), a reduction comparable to that of metformin. In our study, we found that in STZ/high-fat-induced type 2 diabetic rats, Berberine treatment for just 2 weeks significantly improved hyperinsulinemia and insulin resistance, although it did not lead to a significant decrease in blood glucose. This was despite an increase in body weight in Berberine intervention group, which may mean that effects of Berberine do not depend on reduction in body weight. Because Berberine, as a strongly intestinal protector used widely in the treatment of enteritis, is insoluble and rarely absorbed in gastrointestinal tract, it is reasonable to suggest that it may improve diabetes through the modulation of intestinal microbiota and intestinal permeability.

Zhang et al. (2012) studied the alteration of gut microbiota before and after Berberine treatment,
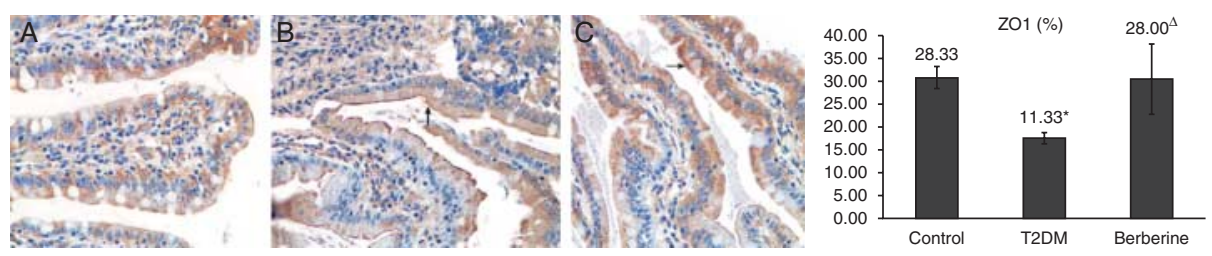

\section{Figure 4}

Immunohistochemical analysis of ZO1 at villous level in ileum mucosa. Type 2 diabetic rats were treated with Berberine (100 mg/kg, Berberine group) or vehicle (T2DM group) for 2 weeks, and nondiabetic rats were used as control group. Expressions of intestinal tight junction protein ZO1 were measured. (A) Healthy control mucosa; (B) diabetic mucosa; extremely http://joe.endocrinology-journals.org DOI: 10.1530/JOE-13-0184
(C) 2013 Society for Endocrinology Printed in Great Britain irregular or absent distribution of labeling at the luminal surface of the intestinal epithelium (arrow). (C) Berberine treatment mucosa: strong cytoplasmic staining; the brush borderline appears similar to healthy mucosa (arrow). ${ }^{*}$ Compared with control group $P<0.05 ;{ }^{\Delta}$ compared with type 2 diabetic group $P<0.05$.

Published by Bioscientifica Ltd 


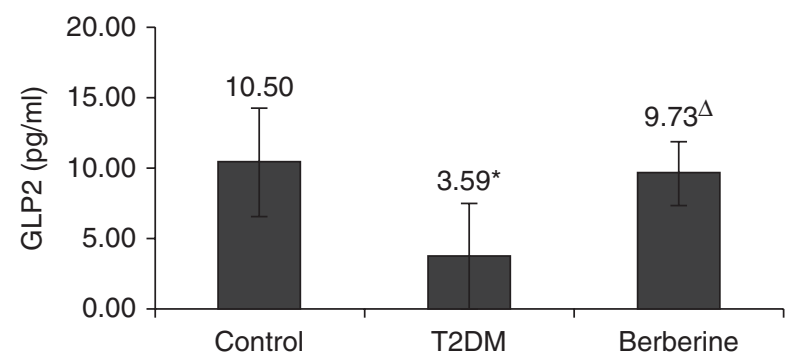

Figure 5

Glutamine-induced GLP2 secretion in ileum tissue. Type 2 diabetic rats were treated with Berberine ( $100 \mathrm{mg} / \mathrm{kg}$, Berberine group) or vehicle (T2DM group) for 2 weeks, and nondiabetic rats were used as control group. Fresh ileum tissue were chopped and transferred to a 24-well plate and stimulated with glutamine (the final concentration was $6.05 \mathrm{mmol} / \mathrm{l}$ ) to induce the secretion of GLP2. *Compared with control group $P<0.05$; ${ }^{\Delta}$ compared with type 2 diabetic group $P<0.05$.

and found a significant alteration of gut microbiota, which was closely associated with host metabolic phenotypes. They also found a few putative short-chain fatty acid (SCFA)-producing bacteria, including Blautia and Allobaculum, that were selectively enriched, along with elevations of fecal SCFA concentrations. Their study implied that the prevention of obesity and insulin resistance by Berberine in high-fat diet-fed rats is at least partially mediated by structural modulation of the gut microbiota. Here in our study, we found that Berberine treatment for only 2 weeks can significantly improve intestinal barrier structure and function in type 2 diabetic rats. Berberine treatment increased the villi/mucosa height and decreased the infiltration of inflammatory cell and lowered the detachment of villi/epithelial cells and therefore can improve the intestinal damage common in diabetic rats. The irregular distribution, fragmentation, and the discontinuity of ZO1 in brush borderline of epithelial cells were also improved in Berberine rats, and the distribution of ZO1 was almost similar to that in healthy mucosa.

The entry of pathogens through the gut via increased intestinal permeability has been proposed to be associated with type 1 diabetes- and insulin resistance-related diseases (Watts et al. 2005, Sapone et al. 2006, Teixeira et al. 2012, Caricilli \& Saad 2013). Recent studies have linked gut microbes and bacterially derived LPS with host innate immune responses, inflammation, and insulin resistance (Cani et al. 2007, 2008). Therefore, our results suggest that the antidiabetic effects of Berberine may depend on its improvement of damaged intestinal mucosa, intestinal permeability, and endotoxemia in diabetic rats.
GLP2 is an enteroendocrine peptide released from intestinal L cells in response to luminal nutrients (Burrin et al. 2001). GLP2 is best known for its role in inducing satiety but is also involved in modulation of intestinal permeability (Moran et al. 2012). GLP2 increases the rate of crypt proliferation, villus elongation, and reduces apoptosis, contributing to an enhanced barrier function (Tsai et al. 1997). GLP2 analogs have been used in the treatment of gastrointestinal-related disorders, such as short bowel syndrome, inflammatory bowel disease, and so on (Wallis et al. 2007, Hornby \& Moore 2011). In our study, we found that Berberine treatment for 2 weeks increases the level of GLP2 in diabetic rats. Correlation analysis found that plasma LPS, ZO1 expression, insulin level, and insulin-resistant index are closely related to GLP2 level. Therefore, our results suggest that the improvement of Berberine on insulin resistance in diabetic rats may partly be explained by amelioration of GLP2 release in ileum, which will consequently improve intestinal permeability, endotoxemia, and inflammation in diabetic rats. Further studies are needed to clarify whether Berberine has a direct effect on GLP2 release or acts indirectly by improving the composition of microbiota in the intestine.

We also found in diabetic rats that glutamine-induced GLP2 secretion is decreased. Although we do not know the exact mechanism, it is clear that the high glucose itself is not the cause as blood glucose did not differ significantly between type 2 diabetic group and Berberine intervention group, whereas GLP2 increased greatly in Berberine group. GLP1 and GLP2 are secreted in a 1:1 ratio by enteroendocrine L cells (Nathanson et al. 2010, Lencioni et al. 2011). Impaired GLP1 secretion is a characteristic of the prediabetic situation such as impaired glucose tolerance and gestational diabetic mellitus (Orskov et al. 1986, Lim $\&$ Brubaker 2006). Therefore, we suggest that impaired GLP2 in prediabetic patients may predispose these patients to T2DM by increasing intestinal permeability and endotoxemia-related inflammation, although we acknowledge that more prospective studies are needed.

In conclusion, in high-fat diet and STZ-induced type 2 diabetic rats, Berberine treatment is efficient in repairing the damaged intestinal mucosa, restoring intestinal permeability and improving endotoxemia, all simultaneously while showing antidiabetic effects and a modulation of GLP2 release in the ileum. The extent to which these individual findings are mechanistically related needs further studies, but they certainly support the hypothesis that Berberine acts via modulation of intestinal function.

Published by Bioscientifica Ltd 


\section{Declaration of interest}

The authors declare that there is no conflict of interest that could be perceived as prejudicing the impartiality of the research reported.

\section{Funding}

This work was supported by the National Nature Science Foundation of China (no. 81273915, 81173428, 81200612).

\section{Author contribution statement}

$\mathrm{C} Y \mathrm{~S}$ and $\mathrm{J} \mathrm{H} \mathrm{Y}$ acquired and analyzed the data and wrote the manuscript. $\mathrm{L} M \mathrm{C}$ and $\mathrm{B} C \mathrm{C}$ conceived the study, analyzed the data, and reviewed the manuscript. $Y K, X Y W, M Y Z, Y G X, Y W$, and $H Z R$ acquired and researched the data. All authors read and approved the final manuscript.

\section{Acknowledgements}

The authors acknowledge the assistance of investigators in this study.

\section{References}

Bäckhed F, Ding H, Wang T, Hooper LV, Koh GY, Nagy A, Semenkovich CF $\&$ Gordon JI 2004 The gut microbiota as an environmental factor that regulates fat storage. PNAS $\mathbf{1 0 1}$ 15718-15723. (doi:10.1073/pnas. 0407076101)

Bi Y, Cai M, Liang H, Sun W, Li X, Wang C, Zhu Y, Chen X, Li M \& Weng J 2009 Increased carnitine palmitoyl transferase 1 expression and decreased sterol regulatory element-binding protein $1 \mathrm{c}$ expression are associated with reduced intramuscular triglyceride accumulation after insulin therapy in high-fat-diet and streptozotocin-induced diabetic rats. Metabolism 58 779-786. (doi:10.1016/j. metabol.2009.01.011)

Burcelin R, Luche E, Serino M \& Amar J 2009 The gut microbiota ecology: a new opportunity for the treatment of metabolic diseases? Frontiers in Bioscience 14 5107-5117. (doi:10.2741/3589)

Burrin DG, Petersen Y, Stoll B \& Sangild P 2001 Glucagon-like peptide 2: a nutrient-responsive gut growth factor. Journal of Nutrition 131 709-712.

Cani PD, Amar J \& Iglesias MA 2007 Metabolic endotoxemia initiates obesity and insulin resistance. Diabetes 56 1761-1772. (doi:10.2337/ db06-1491)

Cani PD, Bibiloni R, Knauf C, Waget A, Neyrinck AM, Delzenne NM \& Burcelin R 2008 Changes in gut microbiota control metabolic endotoxemia-induced inflammation in high-fat diet-induced obesity and diabetes in mice. Diabetes 57 1470-1481. (doi:10.2337/db07-1403)

Cani PD, Possemiers S, Van de Wiele T, Guiot Y, Everard A, Rottier O, Geurts L, Naslain D, Neyrinck A, Lambert DM et al. 2009 Changes in gut microbiota control inflammation in obese mice through a mechanism involving GLP2-driven improvement of gut permeability. Gut $\mathbf{5 8}$ 1091-1103. (doi:10.1136/gut.2008.165886)

Caricilli AM \& Saad MJ 2013 The role of gut microbiota on insulin resistance. Nutrients 5 829-851. (doi:10.3390/nu5030829)

Chou CJ, Membrez M \& Blancher F 2008 Gut decontamination with norfloxacin and ampicillin enhances insulin sensitivity in mice. Nestlé Nutrition Workshop Series. Paediatric Programme 62 127-137. (doi:10.1159/000146256)

Fearnley GR, Vincent CT \& Chakrabarti R 1959 Reduction of blood fibrinolytic activity in diabetes mellitus by insulin. Lancet 21067. (doi:10.1016/S0140-6736(59)91534-X)

Goldfine AB, Conlin PR, Halperin F, Koska J, Permana P, Schwenke D, Shoelson SE \& Reaven PD 2013 A randomised trial of salsalate for

http://joe.endocrinology-journals.org DOI: 10.1530/JOE-13-0184
() 2013 Society for Endocrinology Printed in Great Britain insulin resistance and cardiovascular risk factors in persons with abnormal glucose tolerance. Diabetologia 56 714-723. (doi:10.1007/ s00125-012-2819-3)

Han J, Lin H \& Huang W 2011 Modulating gut microbiota as an anti-diabetic mechanism of Berberine. Medical Science Monitor 17 RA164-RA167. (doi:10.12659/MSM.881842)

Hornby PJ \& Moore BA 2011 The therapeutic potential of targeting the glucagon-like peptide-2 receptor in gastrointestinal disease. Expert Opinion on Therapeutic Targets 15 637-646. (doi:10.1517/14728222. 2011.556620)

Hotamisligil GS, Peraldi P, Budavari A, Ellis R, White MF \& Spiegelman BM 1996 IRS-1-mediated inhibition of insulin receptor tyrosine kinase activity in TNF- $\alpha$ - and obesity-induced insulin resistance. Science $\mathbf{2 7 1}$ 665-668. (doi:10.1126/science.271.5249.665)

Kootte RS, Vrieze A, Holleman F, Dallinga-Thie GM, Zoetendal EG, de Vos WM, Groen AK, Hoekstra JB, Stroes ES \& Nieuwdorp M 2012 The therapeutic potential of manipulating gut microbiota in obesity and type 2 diabetes mellitus. Diabetes, Obesity \& Metabolism 14 112-120. (doi:10.1111/j.1463-1326.2011.01483.x)

Lencioni C, Resi V, Romero F, Lupi R, Volpe L, Bertolotto A, Ghio A, Del Prato S, Marchetti P \& Di Cianni G 2011 Glucagon-like peptide-1 secretion in women with gestational diabetes mellitus during and after pregnancy. Journal of Endocrinological Investigation 34 e287-e290. (doi:10.3275/7799)

Ley RE, Turnbaugh PJ, Klein S \& Gordon JI 2006 Microbial ecology: human gut microbes associated with obesity. Nature 444 1022-1023. (doi:10.1038/4441022a)

Lim GE \& Brubaker PL 2006 Glucagon-like peptide 1 secretion by the L cell. Diabetes 55(Suppl 2) S70-S77. (doi:10.2337/db06-S020)

Membrez M, Blancher F, Jaquet M, Bibiloni R, Cani PD, Burcelin RG, Corthesy I, Macé K \& Chou CJ 2008 Gut microbiota modulation with norfloxacin and ampicillin enhances glucose tolerance in mice. FASEB Journal 22 2416-2426. (doi:10.1096/fj.07-102723)

Moran GW, O’Neill C \& McLaughlin JT 2012 GLP-2 enhances barrier formation and attenuates TNF $\alpha$-induced changes in a Caco- 2 cell model of the intestinal barrier. Regulatory Peptides 178 95-101. (doi:10.1016/ j.regpep.2012.07.002)

Nathanson D, Zethelius B, Berne C, Holst JJ, Sjöholm A \& Nyström T 2010 Reduced plasma levels of glucagon-like peptide- 1 in elderly men are associated with impaired glucose tolerance but not with coronary heart disease. Diabetologia 53 277-280. (doi:10.1007/s00125-0091596-0)

Ni YX 1988 Therapeutic effect of Berberine on 60 patients with type II diabetes mellitus and experimental research. Zhong Xi Yi Jie He Za Zhi 8 711-713.

Ogston D \& McAndrew GM 1964 Fibrinolysis in obesity. Lancet 2 1205-1207. (doi:10.1016/S0140-6736(64)91042-6)

Orskov C, Holst JJ, Knuhtsen S, Baldissera FG, Poulsen SS \& Nielsen OV 1986 Glucagon-like peptides GLP1 and GLP2, predicted products of the glucagon gene, are secreted separately from pig small intestine but not pancreas. Endocrinology 119 1467-1475. (doi:10.1210/endo-1194-1467)

Qin J, Li Y, Cai Z, Li S, Zhu J, Zhang F, Liang S, Zhang W, Guan Y, Shen D et al. 2012 A metagenome-wide association study of gut microbiota in type 2 diabetes. Nature 490 55-60. (doi:10.1038/nature11450)

Sapone A, de Magistris L, Pietzak M, Clemente MG, Tripathi A, Cucca F, Lampis R, Kryszak D, Cartenì M, Generoso M et al. 2006 Zonulin upregulation is associated with increased gut permeability in subjects with type 1 diabetes and their relatives. Diabetes 55 1443-1449. (doi:10.2337/db05-1593)

Soslow RA, Dannenberg AJ, Rush D, Woerner BM, Khan KN, Masferrer J \& Koki AT 2000 Cox-2 is expressed in human pulmonary, colonic, and mammary tumors. Cancer 89 2637-2645. (doi:10.1002/10970142(20001215)89:12<2637::AID-CNCR17>3.0.CO;2-B)

Teixeira TF, Collado MC, Ferreira CL, Bressan J \& Peluzio Mdo C 2012 Potential mechanisms for the emerging link between obesity and 
increased intestinal permeability. Nutrition Research 32 637-647. (doi:10.1016/j.nutres.2012.07.003)

Tremaroli V \& Bäckhed F 2012 Functional interactions between the gut microbiota and host metabolism. Nature 489 242-249. (doi:10.1038/ nature11552)

Tsai CH, Hill M, Asa SL, Brubaker PL \& Drucker DJ 1997 Intestinal growthpromoting properties of glucagon-like peptide-2 in mice. American Journal of Physiology. Endocrinology and Metabolism 273 E77-E84.

Uysal KT, Wiesbrock SM, Marino MW \& Hotamisligil GS 1997 Protection from obesity-induced insulin resistance in mice lacking TNF- $\alpha$ function. Nature 389 610-614. (doi:10.1038/39335)

Wallis K, Walters JR \& Forbes A 2007 Glucagon-like peptide 2-current applications and future directions. Alimentary Pharmacology \& Therapeutics 25 365-372. (doi:10.1111/j.1365-2036.2006.03193.x)

Watts T, Berti I, Sapone A, Gerarduzzi T, Not T, Zielke R \& Fasano A 2005 Role of the intestinal tight junction modulator zonulin in the pathogenesis of type I diabetes in BB diabetic-prone rats. PNAS 102 2916-2921. (doi:10.1073/pnas.0500178102)

Whiting DR, Guariguata L, Weil C \& Shaw J 2011 IDF diabetes atlas: global estimates of the prevalence of diabetes for 2011 and 2030. Diabetes
Research and Clinical Practice 94 311-321. (doi:10.1016/j.diabres.2011. 10.029)

Yan F, Wang L, Shi Y, Cao H, Liu L, Washington MK, Chaturvedi R, Israel DA, Cao H, Wang B et al. 2011 Berberine promotes recovery of colitis and inhibits inflammatory responses in colonic macrophages and epithelial cells in DSS-treated mice. American Journal of Physiology. Gastrointestinal and Liver Physiology 302 G504-G514. (doi:10.1152/ajpgi. 00312.2011)

Yin J, Xing H \& Ye J 2008 Efficacy of Berberine in patients with type 2 diabetes mellitus. Metabolism 57 712-717. (doi:10.1016/j.metabol. 2008.01.013)

Yu Y, Liu L, Wang X, Liu X, Liu X, Xie L \& Wang G 2010 Modulation of glucagon-like peptide-1 release by Berberine: in vivo and in vitro studies. Biochemical Pharmacology 79 1000-1006. (doi:10.1016/j.bcp.2009. 11.017)

Zhang X, Zhao Y, Zhang M, Pang X, Xu J, Kang C, Li M, Zhang C, Zhang Z, Zhang Y et al. 2012 Structural changes of gut microbiota during Berberine-mediated prevention of obesity and insulin resistance in high-fat diet-fed rats. PLOS ONE 7 e42529. (doi:10.1371/journal.pone. 0042529)

Received in final form 14 May 2013

Accepted 11 June 2013

Accepted Preprint published online 11 June 2013 